
$\$$ Research Square
Preprints are preliminary reports that have not undergone peer review.
They should not be considered conclusive, used to inform clinical practice, or referenced by the media as validated information.

\title{
Spontaneous Caesarean scar associated with ectopic tubal twin pregnancy: a case report and review of the literature
}

\section{Ting Zhang}

Affiliated Huai'an No1 People's Hospital of Nanjing Medical University: Huaian First People's Hospital

\section{Fang Wu}

Affiliated Huai'an No1 People's Hospital of Nanjing Medical University: Huaian First People's Hospital

\section{Mi Gong}

Affiliated Huai'an No1 People's Hospital of Nanjing Medical University: Huaian First People's Hospital

Yingchun Gao ( $\nabla$ gaoyingchundoc@163.com )

Affiliated Huai'an No1 People's Hospital of Nanjing Medical University: Huaian First People's Hospital

\section{Case report}

Keywords: caesarean scar pregnancy, ectopic pregnancy, rare condition, therapy, tubal pregnancy

Posted Date: May 6th, 2021

DOI: https://doi.org/10.21203/rs.3.rs-438977/v1

License: (c) (i) This work is licensed under a Creative Commons Attribution 4.0 International License. Read Full License 


\section{Abstract}

\section{Background}

Twin ectopic pregnancy is a rare disease. Herein we report a rare case of spontaneous caesarean scar pregnancy co-existing with tubal pregnancy, and review the relevant literature with respect to treatments.

\section{Case presentation}

A 36-year-old female was admitted to our hospital with complaints of 48 days of menopause and 20 days of vaginal bleeding. Physical examinations and medically assisted examinations suggested the existence of spontaneous caesarean scar pregnancy co-existing with tubal pregnancy. The patient underwent laparoscopic left retrograde salpingectomy and excision of the caesarean scar pregnancy. The patient recovered well during outpatient follow-ups.

\section{Conclusions}

Due to the rarity and particularity of spontaneous caesarean scar associated with ectopic tubal twin pregnancy, accurate diagnosis preoperatively seems particularly difficult. During this report, the possibility of twin ectopic pregnancy should be borne in mind in order to avoid missed diagnosis when meeting women of gestational age who have menopause.

\section{Background}

Ectopic pregnancy refers to the implantation of embryos outside the uterine cavity, and it is one of the most acute and critical gynecological conditions. Caesarean scar pregnancy (CSP) is a specific type of ectopic pregnancy in which the zygote is implanted at the site of a uterine scar caused by a previous cesarean section. The incidence of CSP has increased in China in recent years due to an increased rate of cesarean section, liberalization of the second-child policy, and improvements in transvaginal ultrasound diagnosis in the first trimester [1]. The simultaneous occurrence of caesarean scar pregnancy and tubal pregnancy is extremely rare however, and it may easily lead to missed diagnosis and misdiagnosis. Herein we describe the diagnosis and treatment of a patient admitted to our hospital.

\section{Case Presentation}

The patient was a 36-year-old woman (gravida 5, para 1) who underwent cesarean section at another hospital due to oligohydramnios in 2014. She was hospitalized on 04 March 2020 with the chief complaint of 48 days of menopause and 20 days of vaginal bleeding. Her menstruation was regular, and her last menstruation was on 16 January 2020. Vaginal bleeding occurred without obvious cause on 13 February 2020, accompanied by mild nausea and vomiting. On 22 February 2020 transvaginal pelvic ultrasound revealed that the uterus was enlarged, and a $2.5 \times 0.9 \times 1.8-\mathrm{cm}$ cystic anechoic mass was evident in the lower uterine segment at the scar, with the "double ring sign", a visible yolk sac, and no 
visible germ. The serum human chorionic gonadotropin (HCG) level was $>5000 \mathrm{mlU} / \mathrm{mL}$. On $04 \mathrm{March}$ 2020 transvaginal pelvic ultrasound re-examination depicted enlargement of the uterus, $3.9 \times 2.5-\mathrm{cm}$ cystic anechoic lesions in the lower part of the uterine cavity, the double-ring sign, a visible yolk sac, a germ of approximately $1.0 \mathrm{~cm}$, and cardiac bud pulsation. The germ was approximately $0.7 \mathrm{~cm}$ from the anterior serosal layer. A mixed mass of $4.5 \times 2.9 \mathrm{~cm}$ was observed in the left adnexa, and a cystic anechoic mass of $2.7 \times 1.5 \mathrm{~cm}$ was evident inside it. There was a yolk sac, a germ of approximately 1.0 $\mathrm{cm}$, and cardiac bud pulsation. Ultrasound suggested a possible scar pregnancy, left adnexa mixed mass, and ectopic pregnancy.

The patient was immediately admitted to hospital. After admission she was in a good mental state and her abdomen was flat and soft. Vaginal examination revealed a few dark red vaginal secretions, no lifting pain in the cervix, and mild tenderness in the left adnexal area. Magnetic resonance imaging (MRI) indicated that the anterior wall of the uterus was locally thin, and a gestational sac of approximately $2.6 \mathrm{x}$ $2.2 \mathrm{~cm}$ was evident over it. A round long T1 and long T2 signal of approximately $1.4 \times 2.0 \mathrm{~cm}$ was present in the left adnexal area, and the edge was clear. A few fluid signals were depicted in the uterine and rectal fossa. The diagnosis was scarred uterus, early pregnancy, and abnormal signal in the left adnexal area (Figures 1 and 2). The serum HCG level was 87,584 mIU/mL.

After the relevant examinations were completed, exploratory laparoscopic surgery was planned. A few pelvic adhesions were evident via intraoperative probing. After separation of the adhesions the uterine enlargement was as large as that of the 50th day of pregnancy, and the shape was slightly full. Purplish blue swelling of approximately $4 \times 3 \mathrm{~cm}$ was observed at the scar (Figure 3 ). The other purplish-blue ectopic pregnancy, also approximately $4 \times 3 \mathrm{~cm}$, was located in the ampulla portion of the left fallopian tube (Figure 4). There were no obvious abnormalities in the appearances of the left ovary or right uterine adnexa. Intraoperative diagnoses were pregnancy in the ampulla portion of the left fallopian tube, CSP, scarred uterus, and pelvic adhesion. In view of these findings, a decision was made to perform laparoscopic left retrograde salpingectomy and excision of the CSP. The serum HCG level on the first day after surgery was $2710 \mathrm{mlU} / \mathrm{mL}$. Postoperative histopathology examination results were compatible with CSP and left tubal pregnancy. The postoperative outcome was favorable, and 2 weeks after discharge her serum HCG had decreased to a normal level.

\section{Discussion And Conclusions}

The overall incidence of ectopic pregnancy in the first trimester is approximately $1-2 \%$, and ectopic pregnancy rupture accounts for approximately $2.7 \%$ of pregnancy-related deaths [2]. The most common form of ectopic pregnancy encountered in the clinic is fallopian tube pregnancy. Cervical pregnancy, ovarian pregnancy, CSP, and other locations of ectopic pregnancy are relatively rare. Ectopic twin pregnancy is extremely rare clinically, with an incidence of approximately $1 / 125,000$ reported in the literature. There are comparatively more reports of ectopic twin pregnancy in the same or contralateral fallopian tubes, and it is more commonly seen after assisted reproduction [3,4]. There are also some reports of ovarian ectopic twin pregnancy [5], cornua uteri ectopic twin pregnancy [6], and CSP associated 
with cornua uteri ectopic pregnancy [7]. The current case was particularly rare because it was a spontaneous CSP associated with ectopic tubal twin pregnancy, of which only one case has been reported to date [8]. According to existing reports of ectopic twin pregnancy, CSP lacks specific clinical symptoms compared with other ectopic pregnancies. Menopause, irregular vaginal bleeding, and abdominal pain are the main symptoms, and the diagnosis mainly relies on ultrasound examination. Due to its low incidence, ectopic twin pregnancy can easily be missed or misdiagnosed.

Improper treatment of CSP can lead to massive bleeding, uterine rupture, and uterine resection, the results of which can be life-threatening. Accordingly, CSP attracts a high degree of attention from obstetricians and gynecologists. Early diagnosis, early termination of pregnancy, and early treatment are the primary principles of CSP treatment [1]. To date the precise etiology of CSP remains unclear. Some scholars have proposed a theory involving a uterine incision defect, in which cesarean section causes endometrial injury, incision edge dislocation, or incision infection. Such situations can easily lead to poor healing of a cesarean section incision and a uterine incision defect conducive to zygote implantation. If the endometrium is not decidualized, gestational trophoblast cells can directly invade the myometrium. Studies indicate that independent risk factors for CSP include maternal age $>35$ years, more than three pregnancies (particularly more than five), more than two induced abortions (particularly more than five), < 5 years between pregnancy and previous cesarean section (particularly $<2$ years), and a history of induced abortion after cesarean section [9]. Chen et al. [10] reported that elective cesarean section before labor is an independent risk factor for uterine incision defect after cesarean section. This may be related to poor formation of the lower uterine segment, poor selection of the incision location, poor drainage of uterine blood due to a non-dilated cervix, and/or increased uterine pressure affecting healing of the incision. The present 36-year-old patient's history included a cesarean section in 2014 and three induced abortions, and the current presentation involved her fifth pregnancy. We speculated that the naturally conceived ectopic twin pregnancy may have been related to the patient's history of cesarean section, multiple induced abortions, and pelvic adhesion.

The classification of CSP is closely related to the selection of treatment methods and prognosis, but to date there is no recognized optimal classification method. In a 2016 expert consensus on the diagnosis and treatment of CSP it was classified as type I, II, or III based on the direction of growth of the gestational sac implanted at the scar, and the thickness of the myometrium between the bladder and gestation sac as determined via ultrasound [11]. It is widely accepted that ultrasound is the preferred diagnostic method for CSP, but MRI can clearly depict the relationship between the pregnancy sac and the uterus associated with surrounding organs.

CSP is often mistaken for cervical pregnancy, unavoidable abortion, or incomplete abortion in clinical practice. In the current case B-ultrasonography combined with MRI showed that: (1) a pregnancy sac was partially implanted in the uterine scar and partially located in the uterine cavity; (2) there was obvious deformation of the gestational sac; (3) the myometrium between the pregnancy sac and the bladder was thinned, with a thickness of approximately $7 \mathrm{~mm}$; (4) there was trophoblast blood flow signal in the scar of the uterus; (5) abnormal hypoecho was detected in the left adnexal area, with yolk sac, germ, and 
primitive cardiac tube pulsation. Based on examination results the CSP was considered to be type I associated with left tubal pregnancy.

Treatments for tubal pregnancy mainly include expectant treatment, drug treatment, and surgical treatment. In the present case fetal heart pulsation was observed in the sac in the left fallopian tube, so laparoscopic exploratory surgery was planned. More than 30 CSP treatments have been reported, but the main treatment options include drug therapy (e.g., systemic or local methotrexate therapy), surgical therapy (including uterine curettage, removal of pregnancy and uterine scar repair, hysterectomy), uterine artery embolization (UAE), and combined therapy [12]. Expectant therapy is generally not recommend for CSP. Maheux-Lacroix et al. [13] reported that expectant therapy resulted in $57 \%$ live births, but approximately $63 \%$ of women required a hysterectomy because of placental implantation or uterine rupture in the second trimester. Kaelin Agten et al. [14] investigated 17 CSP patients from two centers and divided them into two groups based on the location that scar pregnancies implanted as determined via first-trimester ultrasound; "on the scar" (group A, 6 cases) and "in the niche" (group B, 11 cases). In group A only one patient underwent cesarean-hysterectomy due to placenta implantation, whereas the other 5 patients delivered via normal cesarean section. In group B 10 patients underwent hysterectomies after cesarean section due to placenta implantation, and 1 patient underwent hysterectomy due to massive hemorrhage at 20 weeks of pregnancy. In further analysis women with a thickness of less than $2 \mathrm{~mm}$ between the musculature of the gestational sac and the bladder in first-trimester ultrasonography were more likely to have placental implantation. If the embryo grows into the scar and protrudes to the bladder the pregnancy needs to be terminated because the outcome of continuing the pregnancy is uterine rupture, or even placenta bladder implantation. The risk and difficulty of the operation increase with gestational age. If the CSP is implanted on the scar, and the muscle layer between the bladder and the embryo is relatively complete, the embryo villus has no obvious muscular infiltration. In such cases it needs to be made clear to the patients and their families that if they choose to continue the pregnancy potentially dangerous placenta previa may occur in the future, which would greatly increase the risk of postpartum hemorrhage and even uterine resection.

Clinical drug therapies for CSP mainly include methotrexate combined with uterine artery embolization, ultrasound-guided local injection of methotrexate into the pregnancy cyst, and systemic injection of methotrexate. Such treatments can fail however, necessitating surgical treatment such as UAE or even hysterectomy for massive bleeding. Some reports indicate that a second treatment is not required if the CSP patient undergoes drug treatment before 7 to 8 weeks of pregnancy because this results in less bleeding, but such patients require a longer recovery time. If medication is used blood flow signals around the pregnancy sac and blood HCG should be detected, and CSP-associated extreme enhanced myometrial vascularity, which is high flow and low-obstruction blood flow around the residual pregnancy sac or placenta, often requires one or more UAEs and even hysterectomy [15]. Blood HCG is assessed once a week, and a reduction of $>15 \%$ each time is considered to indicate effectiveness [11]. For CSP without a yolk sac and fetal heartbeat, ultrasound and blood HCG follow-up can be considered, or systemic methotrexate can be administered [16]. Some studies indicate that the success rate of systemic methotrexate for CSP $(\approx 62 \%)$ is significantly lower than that of tubal pregnancy $(\approx 90 \%)$, which may be 
related to the fact that the fibrous tissue surrounding the implantation of the embryo in the uterine scar reduces the absorption of methotrexate [13].

Uterine curettage can be considered for type I CSP with stable vital signs and a gestational age of less than 8 weeks, but direct uterine curettage is not recommended. Pregnancy should be removed under Bultrasound surveillance or hysteroscopy, and preparations for hemostasis and UAE should be made. There is currently no conclusive evidence of differences between hysteroscopic uterine curettage and ultrasonographic uterine curettage with respect to success rate, intraoperative or postoperative complications, adverse reactions, or $\beta$-HCG normalization time [17]. For type II and III CSP and type I CSP with a gestational age of $>8$ weeks, preventative UAE should be performed before uterine curettage [17]. In a retrospective analysis of 232 women with CSP the success rate of uterine curettage before 7 weeks of pregnancy was approximately $91 \%$, significantly higher than the $56 \%$ success rate of uterine curettage after 7 weeks [18]. Reports suggest that UAE is currently the most widely used CSP treatment, but efficacy and outcomes differ across studies [19]. Studies have also shown that UAE is associated with reduced ovarian function, intrauterine adherence, reduced pregnancy rate, and increased abortion rate [20]. Hence, some scholars have suggested that UAE should only be used in situations in which there is a high risk of bleeding, including older gestational age or ultrasound depicting abundant blood flow or massive vaginal bleeding [21]. The value of UAE for the treatment of CSP requires further study.

For type II and III CSP, surgical resection of pregnancy tissue and scar repair therapy are recommended because of the associated high success rate, rapid recovery, and minimal effect on fertility [20]. The operative methods include traditional transabdominal, laparoscopic, and transvaginal surgery. Studies indicate that surgical resection and defect repair have a high success rate $(\geq 96 \%)$ and entail a low risk of bleeding $(\leq 4 \%)$ [13]. Because of the substantial trauma associated with transabdominal surgery it is generally only used for the emergency treatment of massive bleeding in CSP. Laparoscopic surgery has been widely used because it is associated with less trauma. Above all, laparoscopic surgery requires skilled operators and equipment conditions. If intraoperative bleeding is excessive and hemostasis is difficult to achieve, it must immediately be converted to transabdominal surgery. In 2011 Kang et al. [22] reported transvaginal resection of pregnancy tissue and scar repair for the first time. More than 200 cases of the procedure have since been reported, suggesting that transvaginal surgery is less invasive, has a higher success rate, and is associated with a lower rate of hysterectomy [20]. In the current patient the CSP was complicated by left tubal pregnancy, thus a decision to perform laparoscopic surgery was made, and preparations for blood reserves, UAE, and a transition to transabdominal surgery were also put in place. After intraoperative separation of adhesions, weak tissues were evident at the cesarean scar and a purplish blue swelling of approximately $4 \times 3 \mathrm{~cm}$ was observed. Therefore, we decided to conduct laparoscopic removal of the pregnancy at the uterine scar and uterine scar repair during the operation. At the same time, in order to reduce intraoperative bleeding we injected hypophysis into the uterus to promote contractions. The amount of intraoperative bleeding was approximately $50 \mathrm{~mL}$. The patient recovered well after surgery, and blood HCG returned to a normal level during outpatient follow-up. Because she had no requirement for fertility, we recommended diligent contraception. 
It is difficult to conduct well-designed randomized controlled multicenter CSP studies because of its low incidence. There are no well-established treatment guidelines, and the results of many existing treatments differ across different studies. The present case of spontaneous caesarean scar associated with ectopic tubal twin pregnancy was successfully treated via laparoscopic surgery. The case emphasizes the benefits of early diagnosis and treatment of ectopic pregnancy. During the process of diagnosis and treatment of patients with vaginal bleeding, abdominal pain, and other symptoms ectopic pregnancy should be strongly suspected, and the possibility of twin ectopic pregnancy should be borne in mind in order to avoid missed diagnosis.

\section{Abbreviations}

CSP Caesarean scar pregnancy

HCG Human chorionic gonadotropin

MRI Magnetic resonance imaging

UAE Uterine artery embolization

\section{Declarations}

\section{Acknowledgements}

Not applicable.

\section{Authors' contributions}

Zhang $\mathrm{T}$ reviewed the relevant literature and participated in the drafting of the manuscript. Wu $\mathrm{F}$ participated in the revision of the manuscript. Gong M participated in the information collection. Gao YC is the corresponding author. All authors read and approved the revised manuscript.

\section{Funding}

There is no funding source.

\section{Availability of data and materials}

Not applicable. 


\section{Ethics approval and consent to participate}

Not applicable.

\section{Consent for publication}

Written informed consent for the publication of patient clinical details and clinical images was obtained from the patient.

\section{Competing interests}

The authors declared there were no competing interests.

\section{References}

1. Youbin Hu, Guo H. Progress in diagnosis and treatment of uterine scar pregnancy [J]. Journal of reproductive medicine. 2018;27(07):692-6.

2. Kulp JL, Barnhart KTEctopic. Pregnancy:Diagnosis and Management[J].Women's Health,2008,4(1):79-87.

3. Tsakiridis I, Chatzikalogiannis I, Dagklis T, et al. Spontaneous unilateral twin tubal pregnancy: a rare case report with conservative management[J]. Journal of obstetrics and gynaecology,2019,39(7):1030-1031.

4. JH Pek,,William L,Simpson Jr, et al.LIVE TWIN ECTOPIC PREGNANCY[J].J Emerg Med. 2020 Aug;59(2):e77-e79.

5. Garg MK, Vyas S, Gulati A,et al. Primary twin ovarian pregnancy: case report and review of the literature[J]. J Clin Ultrasound. 2009 Jan;37(1):43-6.

6. J.ERRAZURIZ,A.RACCA,V.SCHUTYSER,et al. Cornual twin pregnancy following IVF/ICSI treatment[J]. Ultrasound Obstet Gynecol. 2018;52:287-8.

7. Li H, Zhao X, Shan S, et al. A case of uterine scar combined with cornual ectopic pregnancy after cesarean section [J]. Chinese Journal of Obstetrics Gynecology. 2017;52(08):564.

8. Zhu J, Shen YY, Zhao YQ, et al. Case Report A case of spontaneous tubal pregnancy with caesarean scar pregnancy[J]. Int J Clin Exp Med. 2014 Sep;15(9):2941-4. 7(.

9. Zhou X, Li H, Fu X. Identifying possible risk factors for cesarean scar pregnancy based on a retrospective study of 291 cases[J]. J Obstet Gynaecol Res. 2020 Feb;46(2):272-8. 
10. Chen $Y$, Han $P$, Wang $Y$, et al. Risk factors for incomplete healing of the uterine incision after cesarean section[J]. Arch Gynecol Obstet,2017,296(2):355-361.

11. Expert consensus on diagnosis and treatment of cesarean scar pregnancy. (2016) [J]. Chinese Journal of Obstetrics and Gynecology, 2016,51 (8): 568-572.

12. Birch Petersen $K$, Hoffmann E, Rifbjerg Larsen $C$, et al. Cesarean scar pregnancy: a systematic review of treatment studies[J]. Fertil Steril. 2016;105(4):958-67.

13. Maheux-Lacroix S, Li F, Bujold E,et al. Cesarean Scar Pregnancies: A Systematic Review of Treatment Options[J]. J Minim Invasive Gynecol. 2017;24(6):915-25.

14. Kaelin Agten A, Cali G, Monteagudo A, Oviedo J, Ramos J, Timor-Tritsch I. The clinical outcome of cesarean scar pregnancies implanted "on the scar" versus "in the niche". Am J Obstet Gynecol. 2017;216:510.e1-6.

15. I. E. TIMOR-TRITSCH.Cesarean scar pregnancy: a therapeutic Dilemma[J].Ultrasound Obstet Gynecol. 2021 Jan;57(1):32-33.

16. Ibrahim A, Abdelazim. Mohannad Abu-Faza,Gulmira Zhurabekova,et al.Successful Pregnancy Outcome Immediately after Methotrexate Treatment for Cesarean Section Scar Pregnancy[J].Gynecol Minim Invasive Ther. 2019 Oct 24;8(4):185-187.

17. Ying L,Huili Zhu,Yuanyuan Hu, et al.Interventions for non-tubal ectopic pregnancy[J].Cochrane Database Syst Rev. 2020 Jul 1;7(7):CD011174.

18. Wu X, Zhang H, Fang X,et al. Factors associated with successful transabdominal sonography-guided dilation and curettage for early cesarean scar pregnancy[J]. Int J Gynecol Obstet, 131(3): 281-284.

19. Ouyang Z, Luo F, Zhong B, et al. Interpretation of guidelines for cesarean scar pregnancy by American maternal and fetal Medical Association [J]. Progress of modern obstetrics Gynecology. 2021;30(01):54-7 + 64 .

20. Ouyang $\mathrm{Z}, \mathrm{Xu} \mathrm{Y,} \mathrm{Li} \mathrm{H,} \mathrm{et} \mathrm{al.} \mathrm{Transvaginal} \mathrm{hysterotomy:} \mathrm{A} \mathrm{novel} \mathrm{approach} \mathrm{for} \mathrm{the} \mathrm{treatment} \mathrm{of}$ cesarean scar pregnancy[J]. Taiwanese J Obstet Gynecol,2019,58(4):460-464.

21. Li H, Guo HY, Han JS, et al. Endoscopic treatment of ectopic pregnancy in a cesarean scar[J]. J Minim Invasive Gynecol. 2011;18:31-5.

22. Kang SY, Park BJ, Kim YW. D.Y. Ro.Surgical management of cesarean scar ectopic pregnancy: hysterotomy by transvaginal approach.Fertil Steril, 96 (2011), pp. e25-e28.

\section{Figures}




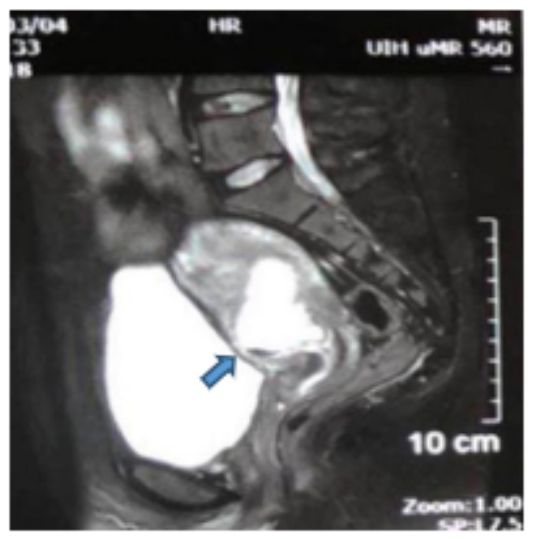

Figure 1

Magnetic resonance imaging depicted thinning of the anterior uterine wall, with a gestational sac of $2.6 \mathrm{x}$ $2.2 \mathrm{~cm}$ at the site of a pre-existing caesarean scar.

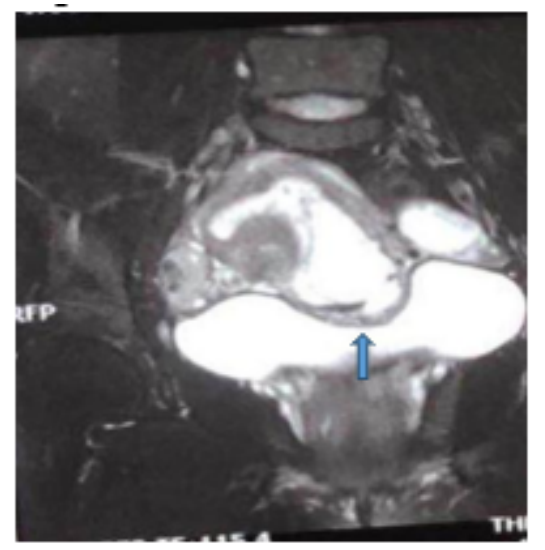

Figure 2

A circular long T1 and long T2 signal shadow of approximately $1.4 \times 2.0 \mathrm{~cm}$ with clear edges in the left adnexal area.

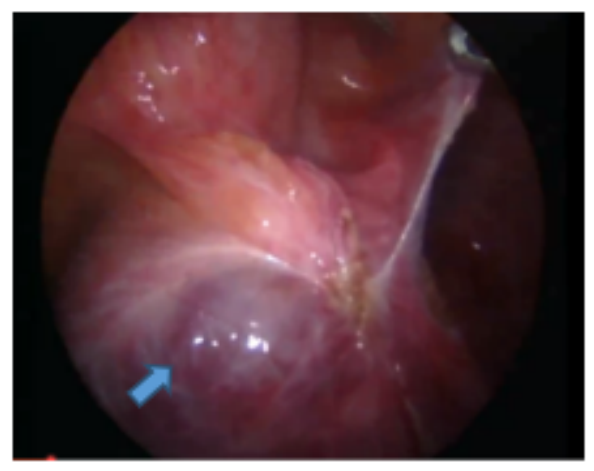

\section{Figure 3}

Image obtained via intraoperative probing depicting a purplish-blue swelling of approximately $4 \times 3 \mathrm{~cm}$ at the site of the scar. 


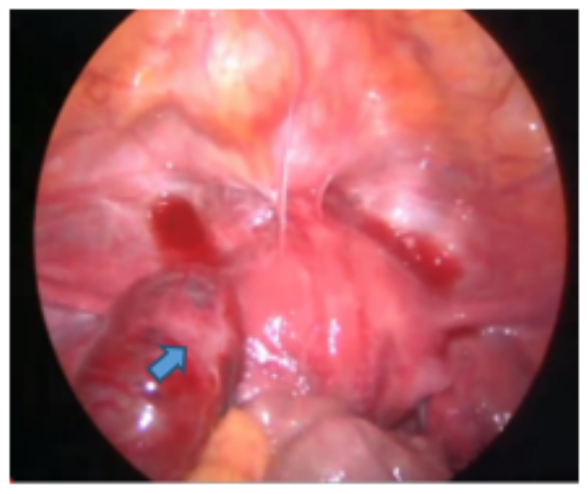

\section{Figure 4}

Image obtained via intraoperative probing depicting a purplish-blue ectopic pregnancy of approximately 4 $x 3 \mathrm{~cm}$ in the ampulla portion of the left fallopian tube. 